\title{
Performance analysis of neuro linguistic programming techniques using confusion matrix
}

\author{
Arun Kumar, Supriya P. Panda \\ Department of Computer Science and Engineering, Faculty of Engineering and Technology, \\ Manav Rachna International Institute of Research and Studies, Faridabad, India
}

\begin{tabular}{l} 
Article Info \\
Article history: \\
Received Aug 27, 2021 \\
Revised Dec 24, 2021 \\
Accepted Jan 14, 2022 \\
\hline Keywords: \\
Confusion matrix \\
Neuro lingustic programming \\
Qualitative sentiemnt analysis \\
Swish pattern \\
Visual kinesthetic dissociation
\end{tabular}

\begin{abstract}
During numerous qualitative surveys, swish patterns and visual kinesthetic dissociation (V/KD) were employed to examine attitudes and past occurrences. Neuro-linguistic programming (NLP) workshops in both hypnotic and non-hypnotic experimental sessions were held for forty days. Results demonstrated that negative sentiments and various emotional factors were significantly higher in 10-days' workshop sessions as compared to 40 days' sessions. Following the qualitative sentiments recollection, NLP workshops with various activities in the fear and stress indexing segment were increased in length. The NLP procedure was followed by the decreased negative emotional intensity in both groups; also, the results have been improved when using swish patterns and V/KD techniques. The performance analysis shows the results of improving emotional and sentimental factors in various NLP workshops. The workshops ranged in length from five to forty days. The specifications for workshops were selected based on the human mind's pre-determined conditions. The performance factors of two significant NLP techniques used in NLP workshops were compared and both techniques' performance factors were found to be adequate in terms of modifying behavior patterns. Using the confusion matrix, the overall accuracy percentage between V/KD and swish patterns is calculated, and an increase from 0.65 to 0.83 in the stressed parameters is shown.
\end{abstract}

This is an open access article under the CC BY-SA license.

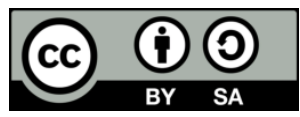

\section{Corresponding Author:}

Arun Kumar

Departmentof Computer Science and Engineering, Faculty of Engineering and Technology

Manav Rachna International Institute of Research and Studies

Sector 43, Aravalli Hills, Delhi-Surajkund Road, Faridabad, Haryana, India

Email: scorearun84@gmail.com

\section{INTRODUCTION}

Neuro-linguistic programming (NLP) is a pseudoscientific approach to explore the human mind. The human mind relates to the neurological system. The neurological system helps the human mind to receive information through the external environment and make it resourceful by using various senses. The ability to do any task in everyday life depends upon the responses collected by the nervous system [1]. Neuro concerns with the neurological system. The NLP is based on the fact that sensory information can be convertedinto a thought process. Thought processes activate the neurological system, which affects physiology, emotions, and behavior [2], [3]. The combination of various sentiments and emotions makes the behavioral patterns appropriate that can fulfill the requirement of internal as well as external environments [4]. Linguistics is the study of the language used to express how people feel about the world. According to NLP, human senses are responsible for the creation of emotions, feelings, and a variety of characteristics of communicated information. Linguistics is related to the languages that are used in the real world to describe 
feelings and attitudes. Among them are the kinesthetic, visual, auditory, olfactory, and gustatory senses [5], [6]. These senses can help the human mind to filter the content in terms of various sentiments like attitude, memories, sorting, values, beliefs, and strategies [7], [8]. The combination of senses and sentiments can be used to produce changing behavioral patterns depending upon the various emotional factors [9], [10]. The two above terms 'Neurons' and 'Linguistics' combined to produce the programming patterns for the human mind. In many research, it has been notified that the neurological patterns of the human mind can be changed according to the various environmental conditions [11]. Every time neurons of the mind make the programming patterns depending upon these environmental conditions [12]-[14]. The internal as well as external conditions are more resourceful and can be used to analyze various behavioral patterns of the mind [15]. In the remainder of this paper, section 2 focuses on research method; section 3 examines the proposed framework and architecture as how this model will help to analyze the realistic patterns of the mind when conducted qualitative surveys in various NLP workshops. In section 4, preprocessing results and NLP workshop results using swish pattern are discussed. The conclusion is stated in section 5 as the overall accuracy percentage between visual kinesthetic dissociation (V/KD) and swish patterns, which is quantified using the confusion matrix and seen to improve from $65 \%$ to $83 \%$ in the stressed parameters.

\section{RESEARCH METHOD}

Richard Bandler in 1985 introduced the swish pattern is the modified version among various neuro linguistic programming techniques [9]. The swish pattern provides real insight into areas such as sub-modalities and multiple perspectivesto break the linkage of thoughts related to current behavior patterns and replace it with the appropriate ones. Patterns such as smoking cessation, speaking, selfesteem, self-confidence, and nervousness can be the breakage patterns of the current behavior pattern [10], [11]. The swish pattern is a well-known NLP technique that is frequently used to assist people break difficultto-break habitual behaviours. Here are some important terms used in research methods of NLP [12].

\subsection{NLP techniques}

It is important to know that NLP is rooted in some very important presuppositions on which its techniques, meta-tactics, are based. For our purpose, NLP presupposes that Map is not the territory. The representations we use to organize our experience of the world are nut the world. They are neurological transformations that may or may not be accurate. As human beings, we input, output, and process information about the territory around us. That information is coded in terms of the five sensory systems: visual, auditory, kinesthetic, olfactory, and gustatory [16]. Simply put, the subjective experience "is generated as an interaction between what the external world provides for human senses to take in, and what our minds produce in the way of internally-generated imagery, internal dialogue, smells, and feelings [17].

\subsection{Swishing}

A strategy for removing a negative input is swishing. Swish, according to Bandler and Grinder, is the act ofbreaking a mental pattern that leads to unwanted behavior and replacing it with one that leads to desired behavior (1985). This entails identifying a 'cue' associated with the undesirable behavior. Swishing also includes aspects for changing the behavioral patterns, hitting the negative points, and then calculating the results of improvement in various NLP workshops [18].

\subsection{Visual kinesthetic dissociation (V/KD)}

In this study, the $\mathrm{V} / \mathrm{KD}$ technique is used to express the emotional factor of human beings in terms of their feelings, emotions, and sentiments. V/KD is useful to change the behavioral patterns of the human mind. It's being used by NLP trainers while conducting NLP workshops [19].

\subsection{Problem statement}

In various social communities, people and working professionals are always facing difficulty in dealing with behavioral patterns when communicating with each other. Web communications always deal with various online chats and messages like on Twitter and Facebook. Text messages and chats have very limited understanding on web platforms. In this regard, the quantitative approach in sentiment analysis has less impact in dealing with changing behavioral patterns. The main impact on the qualitative approach with NLP techniques is the aim of this research.

\subsection{Problem solution}

To overcome the above problem, neuro-linguistic programming (NLP) has been introduced in previous studies [10]. The fact that NLP is "simply common sense" might be considered as strength, and common sense can be codified to make it more accessible. Various NLP techniques as V/KD and swish 
patterns have been used to resolve the problem that arises in the quantitative approach of sentiment analysis. The main impact on the qualitative approach with NLP techniques by calculating the overall accuracy percentage between $\mathrm{V} / \mathrm{KD}$ and swish patterns is quantified using the confusion matrix and seen to improve from $65 \%$ to $83 \%$ in the stressed parameters.

\section{PROPOSED MODEL}

Neuro linguistic programming (NLP) provides a way to change the behavioral patterns among various social communities. Figure 1 is the modified version of NLP model based on the data sets and implementation results shown in previous studies. The structure of this model representsdata collection methods, preprocessing techniques used in various NLP Workshops. This model will be used to find the latest findings and compare both $\mathrm{V} / \mathrm{KD}$ and swish pattern. For both strategies, the confusion matrix serves as a performance indicator. Performance analysis, sentiment detection graph, confusions matrix findings will be used as predictive results for the proposed model. This model will help to analyze the realistic patterns of the mind when conducted qualitative surveys in various NLP workshops.

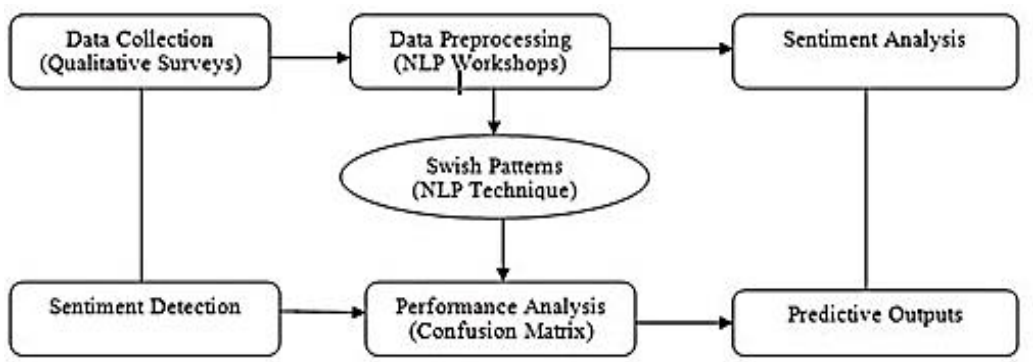

Figure 1. Proposed model

\section{RESULTS AND DISCUSSIONS}

The performance and results of NLP workshops in terms of confusion matrix is shown in Tables 1 and 2 by using V/KD and swish pattern, respectively. As discussed earlier, NLP workshops conducted to show the realistic pattern of mind where the participants have gone through the workshops for duration of 0 to 40 days [17], [18]. The fearful mind is always stressed as a result it can itself create negative patterns [19]-[21]. To avoid these circumstances participants have been given the practice instructions as a medicine to maintain the consistency of the positive patterns. Tables 1 and 2 show the results predicted by visual kinesthetic dissociation (V/KD) technique used in NLP. Table 3 will be taken as sample to find the inconsistent patterns of the mind recognized in NLP workshops while applying swish pattern [22]-[24]. Swish patterns and its sample can be converted into computational language. Machine learning techniques like confusion matrix can be used to find the uncertainty in various patterns of the mind [25], [26]. The dataset of NLP workshops can be considered as sample data of resultant features and parameters [27]. The qualitative research by using NLP contains dataset of 880 participants. Participants have been divided in various categories and NLP techniques like V/KD and swish patterns are the main source of these surveys and workshops.

Table 1. NLP workshop results using V/KD

\begin{tabular}{cccc}
\hline NLP Testee & NLP Trainee Duration & Actual Negative \% & Positive Negative Sentiment Correction \\
\hline Testee \# 1 & $0-5$ & $80 \%$ & $14 \%$ \\
Testee \# 2 & $5-10$ & $85 \%$ & $40 \%$ \\
Testee \# 3 & $10-15$ & $85 \%$ & $55 \%$ \\
Testee \# 4 & $15-20$ & $90 \%$ & $65 \%$ \\
Testee \# 5 & $20-25$ & $90 \%$ & $70 \%$ \\
Testee \# 6 & $25-30$ & $90 \%$ & $75 \%$ \\
Testee \# 7 & $35-40$ & $90 \%$ & $80 \%$ \\
\hline \multicolumn{4}{c}{ Overall (Accuracy NLP (V/KD)) =Actual Sentiment Detection/Predicted Correction $=0.65$}
\end{tabular}

Sentiment detection graph between positive and negative sentiment has been shown in Figure 2. Graph is implemented through Python (Spyder). Figure 3 shows implementation of code to improve the quality of sentiments in Python. 
Table 2. NLP workshop results using swish pattern

\begin{tabular}{cccccc}
\hline $\begin{array}{c}\text { NLP } \\
\text { Testee }\end{array}$ & $\begin{array}{c}\text { NLP Trainee } \\
\text { Duration }\end{array}$ & $\begin{array}{c}\text { Actual }(-\mathrm{ve}) \\
\text { Sentiment } \%\end{array}$ & $\begin{array}{c}\text { Confusion Matrix } \\
\text { Category }\end{array}$ & $\begin{array}{c}\text { Predicted (-ve) } \\
\text { Sentiment } \%\end{array}$ & $\begin{array}{c}\text { Confusion Matrix } \\
\text { Category }\end{array}$ \\
\hline Testee\#1 & $0-10$ & 55 & True Positive & 45 & True(+ve) \\
Testee\#2 & $10-20$ & 55 & True Positive & 50 & True(+ve) \\
Testee\#3 & $20-30$ & 100 & False Negative & -ve (Failed) & False(-ve) \\
Testee\#4 & $30-40$ & 60 & True Positive & 40 & True(+ve) \\
Testee\#5 & $0-10$ & 30 & True Positive & 70 & True(+ve) \\
Testee\#6 & $10-20$ & 55 & True Positive & 45 & True(+ve) \\
Testee\#7 & $20-30$ & 35 & True Positive & 65 & True(+ve) \\
Testee\#8 & $30-40$ & 40 & True Positive & 90 & True(+ve) \\
Testee\#9 & $0-10$ & 75 & True Positive & 25 & True(+ve) \\
Testee\#10 & $10-20$ & 10 & True Positive & 90 & True(+ve) \\
Testee\#11 & $20-30$ & 15 & True Positive & 85 & True(+ve) \\
Testee\#12 & $30-40$ & -20 & False Negative & 80 & False(-ve) \\
\hline
\end{tabular}

Table 3. Conversion of swish pattern results (confusion matrix)

\begin{tabular}{ccc}
\hline S.No. & Actual Inputs & Predicted Results \\
\hline 1 & 1 & 1 \\
2 & 1 & 1 \\
3 & 0 & 0 \\
4 & 1 & 1 \\
5 & 0 & 0 \\
6 & 1 & 1 \\
7 & 0 & 1 \\
8 & 0 & 0 \\
9 & 1 & 1 \\
10 & 0 & 0 \\
11 & 1 & 0 \\
12 & 0 & 0 \\
\hline
\end{tabular}

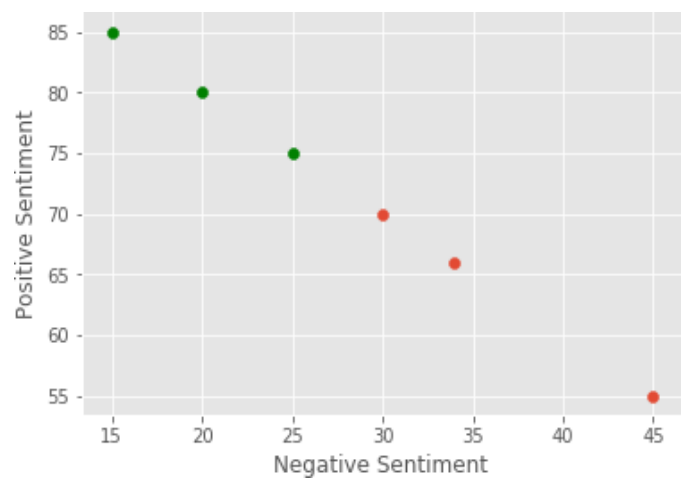

Figure 2. NLP sentiment detection graph

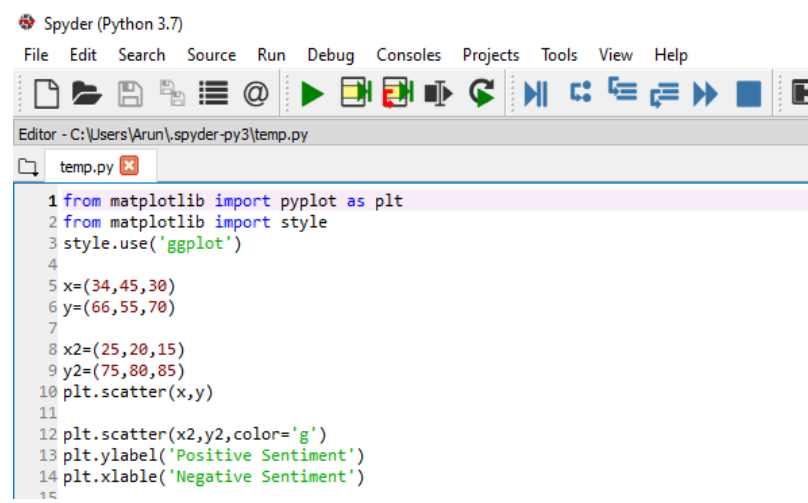

Figure 3. Spyder (Python3.7) snippet

The above graph shows the confusion matrix factors explained in Table 3. Figure 4 shows the implementation of Table 3 in Python. Table 3 is conversion of realistic pattern of NLP workshop results and can be converted and implemented as per confusion matrix graph. In confusion matrix, the true positive (TP) rated as 5 means the actual results as per the predictions are true. The next parameter of the confusion matrix is true negative (TN) rated as 5 means the results are matching and touch the boundary conditions of the predicted conditions. The false positive (FP) and false negative (FN) rated as 1. The graph has been implemented in Python language with pandas and matplotlib libraries. Table 4 shows the accurate results based on the above confusion matrix graph as shown in Figure 4.

Table 4. NLP accuracy results using confusion matrix

\begin{tabular}{ccc}
\hline $\mathrm{N}=12$ & Predicted: NO & Predicted: YES \\
\hline Actual: NO & $\mathrm{TN}(5)$ & $\mathrm{FP}(1)$ \\
Actual: YES & $\mathrm{FN}(1)$ & $\mathrm{TP}(5)$ \\
\hline \multicolumn{2}{c}{ Overall Accuracy: $\mathrm{TP}+\mathrm{TN} / \mathrm{TP}+\mathrm{TN}+\mathrm{FP}+\mathrm{FN}=0.83$}
\end{tabular}




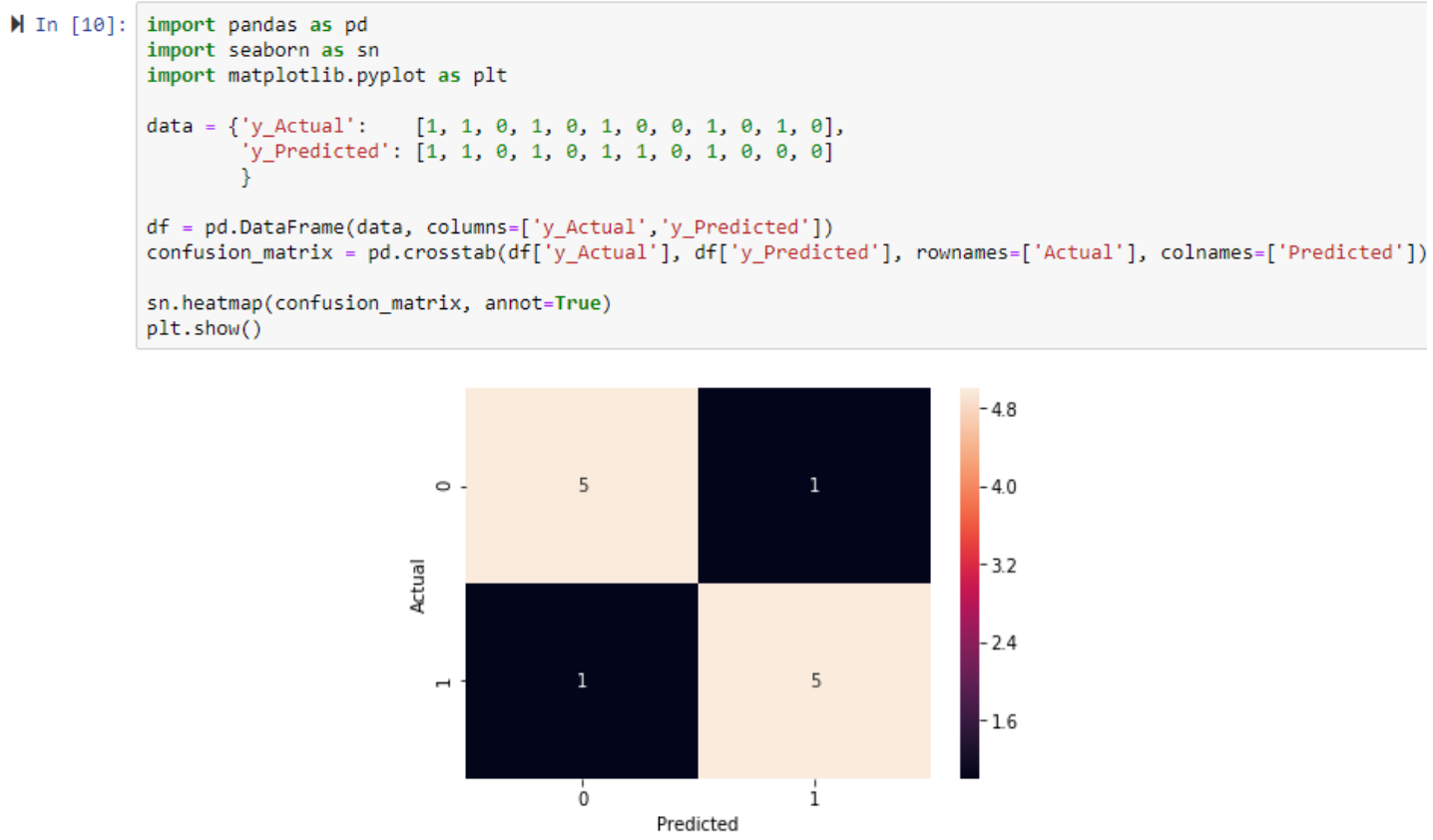

Figure 4. Confusion matrix graph (Python 3.7) Snippet

Figure 5 shows the results and calculations of confusion matrix for sample of data used in Table 3. The sample of total populations is 12. In which half of the sample considered as the positive and other half considered negative. The positive test cases are considered to be same as negative test cases to maintain the consistency level of swish pattern used in NLP workshop.

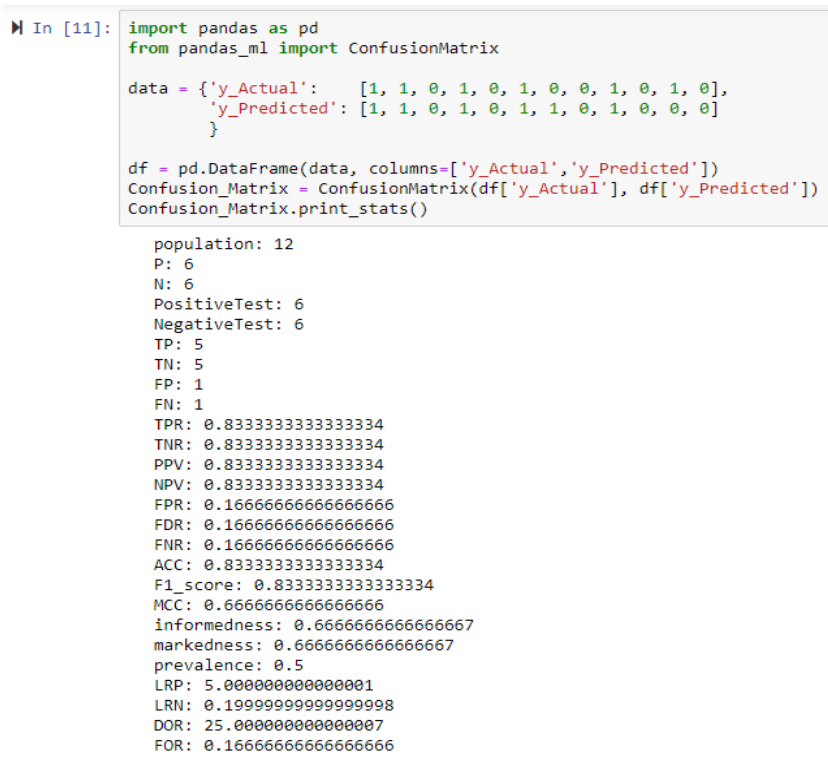

Figure 5. Confusion matrix implementation (Python) snippet

The main resultant factors of confusion matrix are true positive, true negative, false positive, and false negative. The above results showing the accuracy level of 0.83 for the swish pattern technique used in NLP workshops. As the Figure 6 and Figure 7 differentiate the accuracy levels for V/KD and swish patternsthathave been used for the NLP workshops. The resultant factors have been improved from 0.65 to 0.83 even if the consistency level down in between 10 to 15 days. 


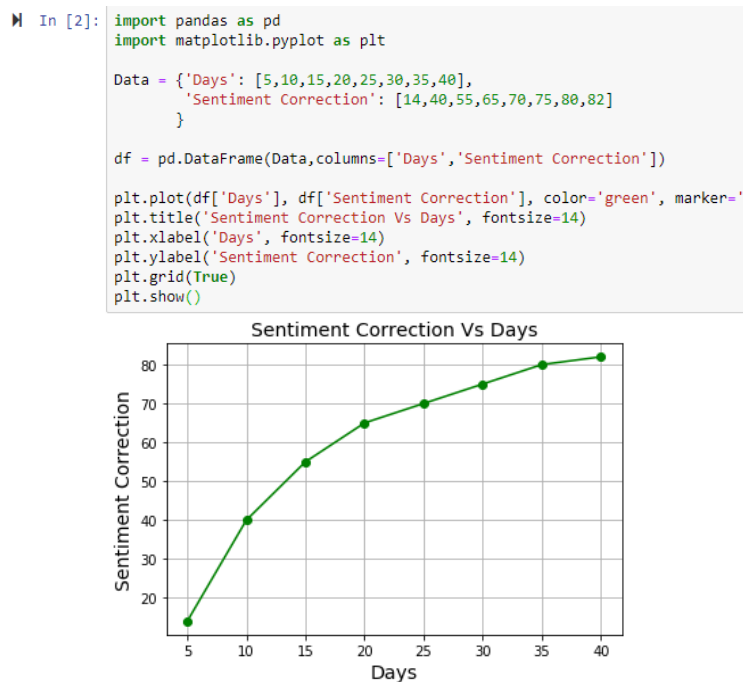

Figure 6. V/KD performance (Python) snippet

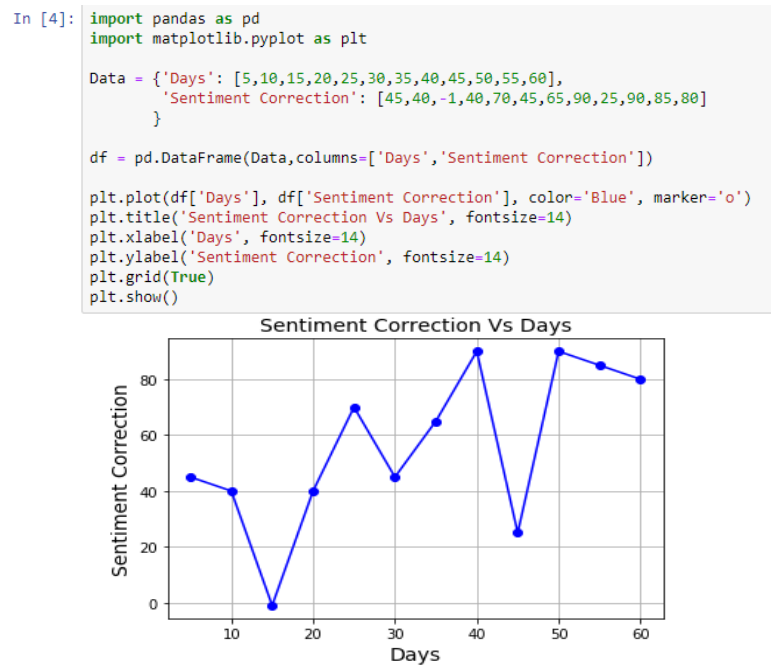

Figure 7. Swish pattern performance (Python) snippet

\section{CONCLUSION}

NLP techniques have been applied in these workshops on the human mind to change the behavioral patterns according to various parameters of the human mind. The workshops durations have been decided from 5 to 40 days. In NLP workshops, parameters have been decided according to the pre-defined conditions of the human mind. The human mind pattern can always be changed depending upon the environmental factors in external world criteria. The results show the comparison between two important NLP techniques used in NLP workshops and the performance factors of both the techniques has been shown satisfactory in terms of changing behavior patterns. This Research shows the 10 days' results of the improved factors as well as 40 days' results of the improved factors. The overall accuracy percentage between $\mathrm{V} / \mathrm{KD}$ and swish patterns is quantified using the confusion matrix and seen to improve from 0.65 to 0.83 in the stressed parameters.

\section{FUTURE SCOPE}

As a result of the numerous performance elements used in NLP courses, human thought patterns can be modified in as little as 40-60 days. With the use of numerous case studies in NLP workshops, it may be possible to cut the number of days. NLP strategies such as reframing the model will be beneficial in expanding the scope and producing solutions with the fewest conceivable outcomes. In the future, the modeling technique will be useful in a variety of case studies and hypothetical situations.

\section{REFERENCES}

[1] K. Z. Ahmad, "Improving Emotional Intelligence (EI) using Neuro Linguistic Programming (NLP) techniques," International Conference on Advances in Business, Management and Law (ICABML) 2017, vol. 1, no. 1, pp. 172-183, Dec. 2017, doi: 10.30585/icabml-cp.v1i1.19.

[2] M. H. Amirhosseini and H. Kazemian, "Automating the process of identifying the preferred representational system in Neuro Linguistic Programming using Natural Language Processing," Cognitive Processing, vol. 20, no. 2, pp. 175-193, Mar. 2019, doi: $10.1007 / \mathrm{s} 10339-019-00912-3$.

[3] R. Pishghadam and S. Shayesteh, "Neuro-linguistic Programming (NLP) for Language Teachers: Revalidation of an NLP Scale," Theory and Practice in Language Studies, vol. 4, no. 10, Oct. 2014, doi: 10.4304/tpls.4.10.2096-2104.

[4] A. M. Alhassan and W. M. N. W. Zainon, "Brain tumor classification in magnetic resonance image using hard swish-based RELU activation function-convolutional neural network," Neural Computing and Applications, Jan. 2021, doi: 10.1007/s00521020-05671-3.

[5] G. E. Florina, G. V. Teodor, P. S. Cornelia, and D. Marin, "Neurolinguistic Programming and the Relationship between Attention and Anxiety in Alpine Skiing Juniors," Procedia - Social and Behavioral Sciences, vol. 191, pp. 1634-1638, Jun. 2015, doi: 10.1016/j.sbspro.2015.04.577.

[6] R. Gaspar, C. Pedro, P. Panagiotopoulos, and B. Seibt, "Beyond positive or negative: Qualitative sentiment analysis of social media reactions to unexpected stressful events," Computers in Human Behavior, vol. 56, pp. 179-191, Mar. 2016, doi: 10.1016/j.chb.2015.11.040.

[7] B. P. Gibson, The complete guide to understanding and using NLP: neuro-linguistic programming explained simply. Ocala, Fla.: Atlantic Pub. Group, 2011.

[8] G. Emilia Florina et al. "Neuro-linguistic Programming based on the Concept of Modelling," Procedia - Social and Behavioral Sciences, vol. 116, pp. 3693-3699, Feb. 2014, doi: 10.1016/j.sbspro.2014.01.825. 
[9] Autor: John Grinder and R Frank Pucelik, The Origins of neuro linguistic programming. Editora: Carmarthen England: Crown House Pub, 2013

[10] E. Hosseinzadeh and A. Baradaran, "Investigating the Relationship between Iranian EFL Teachers' Autonomy and Their NeuroLinguistic Programming,” English Language Teaching, vol. 8, no. 7, Jun. 2015, doi: 10.5539/elt.v8n7p68.

[11] A. Lashkarian and S. Sayadian, "The Effect of Neuro Linguistic Programming (NLP) Techniques on Young Iranian EFL Learners' Motivation, Learning Improvement, and on Teacher's Success," Procedia - Social and Behavioral Sciences, vol. 199, pp. 510-516, Aug. 2015, doi: 10.1016/j.sbspro.2015.07.540.

[12] Y. Javadi and S. A. Asl, "Neuro-linguistic Programming, Teacher's Identity, and Teachers' Effectiveness," Journal of Language Teaching and Research, vol. 11, no. 3, p. 389, May 2020, doi: 10.17507/jltr.1103.07.

[13] G. A. Juhnke, K. M. Coll, M. F. Sunich, and R. R. Kent, "Using a Modified Neurolinguistic Programming Swish Pattern With Couple Parasuicide and Suicide Survivors," The Family Journal, vol. 16, no. 4, pp. 391-396, Oct. 2008, doi: $10.1177 / 1066480708322807$.

[14] M. Karunaratne, "Neuro-linguistic programming and application in treatment of phobias," Complementary Therapies in Clinical Practice, vol. 16, no. 4, pp. 203-207, Nov. 2010, doi: 10.1016/j.ctcp.2010.02.003.

[15] M. S. Keezhatta, "The Impact of Neuro-Linguistic Programming on English Language Teaching: Perceptions of NLP-Trained English Teachers," International Journal of English Linguistics, vol. 9, no. 6, p. 454, Nov. 2019, doi: 10.5539/ijel.v9n6p454.

[16] İ. Kök, "Listening Comprehension Achievement and Brain Dominance," Procedia - Social and Behavioral Sciences, vol. 122, pp. 329-334, Mar. 2014, doi: 10.1016/j.sbspro.2014.01.1348.

[17] J. Walter, "Neurolinguistic programming: verbal communication," BMJ, vol. 326, no. 7389, pp. 83S83, Mar. 2003, doi: 10.1136/bmj.326.7389.s83.

[18] Y. Kotera and W. Van Gordon, “Japanese managers' experiences of neuro-linguistic programming: a qualitative investigation," The Journal of Mental Health Training, Education and Practice, vol. 14, no. 3, pp. 174-185, May 2019, doi: 10.1108/jmhtep-06-2018-0033.

[19] E. Rogozinska, "Neuro-linguistic programming for teaching and learning," The Journal of Linguistic and Intercultural Education, vol. 9, no. 2, pp. 149-160, Oct. 2016, doi: 10.29302/jolie.2016.9.2.12.

[20] T. Kovalevska and A. Kovalevska, "Utilizing the Neurolinguistic Programming Technologies in Foreign Languages Teaching Practice in Ukrainian Universities," SSRN Electronic Journal, 2020, doi: 10.2139/ssrn.3735652.

[21] A. Lashkarian and S. Sayadian, "The Effect of Neuro Linguistic Programming (NLP) Techniques on Young Iranian EFL Learners' Motivation, Learning Improvement, and on Teacher's Success," Procedia - Social and Behavioral Sciences, vol. 199, pp. 510-516, Aug. 2015, doi: 10.1016/j.sbspro.2015.07.540.

[22] J. Neidhardt, N. Rümmele, and H. Werthner, "Predicting happiness: user interactions and sentiment analysis in an online travel forum," Information Technology \& Tourism, vol. 17, no. 1, pp. 101-119, Mar. 2017, doi: 10.1007/s40558-017-0079-2.

[23] A. D. Poernomo and S. Suharjito, "Indonesian online travel agent sentiment analysis using machine learning methods," Indonesian Journal of Electrical Engineering and Computer Science, vol. 14, no. 1, p. 113, Apr. 2019, doi: 10.11591/ijeecs.v14.i1.pp113-117.

[24] R. S. Nompo, A. Pragholapati, and A. L. Thome, "Effect of Neuro-Linguistic Programming (NLP) on Anxiety: A Systematic Literature Review,” KnE Life Sciences, pp. 496-507, Mar. 2021, doi: 10.18502/kls.v6i1.8640.

[25] G. Roderique-Davies, "Neuro-linguistic programming: cargo cult psychology?," Journal of Applied Research in Higher Education, vol. 1, no. 2, pp. 58-63, Jul. 2009, doi: 10.1108/17581184200900014.

[26] H. S. Rugo et al., "Prevention of everolimus-related stomatitis in women with hormone receptor-positive, HER2-negative metastatic breast cancer using dexamethasone mouthwash (SWISH): a single-arm, phase 2 trial," The Lancet Oncology, vol. 18, no. 5, pp. 654-662, May 2017, doi: 10.1016/s1470-2045(17)30109-2.

[27] N. Moslemi and P. Habibi, "The Relationship among Iranian EFL Teachers' Professional Identity, Self-Efficacy and Critical Thinking Skills," HOW, vol. 26, no. 1, pp. 107-128, Jan. 2019, doi: 10.19183/how.26.1.483.

\section{BIOGRAPHIES OF AUTHORS}

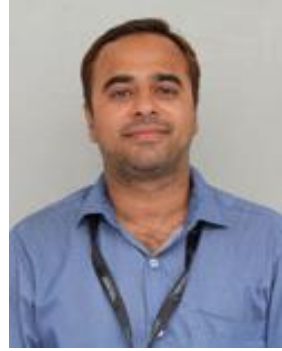

Arun Kumar (M. Tech, Ph. D (Pursuing) (D) SC SC P is currently pursuing Ph. D and working as an Assistant Professor at Manav Rachna International Institute of Research \& Studies, Faridabad, India. He has over 11 years of experience in Academics and Research and published in various National and International Journals. He can be contacted at email: scorearun84@gmail.com.

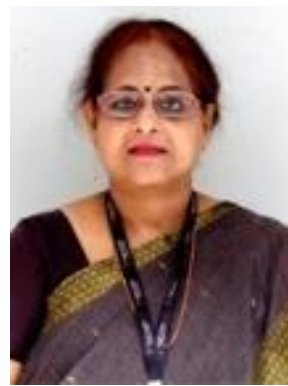

Supriya P. Panda (MS, Ph. D (BGSU, Ohio, USA), (D) SC SC is working as a professor (CSE) and HoD (CSE) at Manav Rachna International Institute of Research \& Studies at Faridabad (India) since 2016. She started her stint at BITS, Pilani and Rajasthan, India wherein she served for ten years. She has over three decades of experience in academics across various organizations in India and abroad. She has numerous research papers in reputed national and International Journals to her credit. She can be contacted at email: supriya.fet@mriu.edu.in. 